\title{
Total laparoscopic billroth-1(Distal) gastrectomy compare(TLDG) To open billroth-1 gastrectomy(ODG) in early gastric cancer
}

\author{
Alhadhrami B*, Kinoshita T, Gotohda N, Kato Y, Shibasaki H, Tkahashi S, and Konishi M \\ Royal Hospital, Muscat, Oman
}

\begin{abstract}
Objective: TLDG (using the delta-shaped anastomosis) in the treatment of early gastric cancer is well accepted procedure in Japan. Conventional open Billorth-1 gastrectomy still mainly performed because of technical difficulties, especially intracorporeal gastroduodenostomy. Efficacy \& feasibility of this procedure compared to open procedure to be elicited.

Methods: Case-match retrospective study analysis of single institute's experience in National Cancer Center East, Chipa, Japan. TLDG between Aug 2010 and Jul 2012. This procedure was compared with ODG as control between Aug 2008 and Jul 2010. 100 patients in each group, case-matched with staging and gender. Age for TLDG was $62.9( \pm 11.1)$ and $65.4( \pm 10.7)$ for ODG. M: F ratio in each group was $62: 38$. Early gastric cancer was considered for any patient with stage 1 a, 1 b \& 2a. BMI for the first group was $22.5( \pm 2.6)$ and second group $22.6( \pm 3)$.

Outcomes: Operation time, Blood loss, number of harvested lymph node, postoperative hospital stay, clinical pathway, postoperative complications.

Results: No difference in the mean age, BMI, gender. Significant differences $(\mathrm{P}<0.001)$ present between the two groups in blood loss, operation time $\&$ postoperative hospital stay. Estimated blood loss for TLDG group was $17.9( \pm 21.8) \mathrm{ml}$ and ODG was $234( \pm 257.7) \mathrm{ml}$. Operation time for TLDG was $173( \pm 30.5) \mathrm{min}$. and ODG was 204 ( \pm 42.7). Post-operative hospital stay for TLDG was $8.5( \pm 2.4)$ Days and for the ODG 11.2 ( \pm 9.5$)$. No conversion from laparoscopy to open surgery. No difference in complication rate between two groups.

Conclusion: When skilled surgeon is available TLDG is more efficient and feasible compare to open distal gastrectomy. Significant reduction in blood loss, postoperative hospital stay \& operation time can be gained in TLDG, and contribute to the patients' quality of life. This study has limitations of retrospective study; therefore a prospective study should be conducted in near future.
\end{abstract}

\section{Introduction}

Stomach cancer still one of the common malignancies in the world, there were estimated to be 988,000 cases of stomach cancer diagnosed worldwide in 2008 [1]. Stomach Cancer still is one of the commonest cancers In Japan. Many Clinical Trials have shown that laparoscopic procedures compared to open procedures are safe and efficient. In Japan, Billroth-1 has been long preferred by many surgeons as reconstruction procedure after distal gastrectomy because of the physiological advantages of allowing food to pass through the duodenum [2-7]. Also, this type of reconstruction make the endoscopic surveillance much easier and faster.

Total Laparoscopic Billroth-1 Gastrectomy (using the deltashaped anastomosis) in the treatment of early gastric cancer is becoming more popular \& accepted procedure in Japan. However, in the world, conventional open Billorth-1 gastrectomy still mainly performed because of technical difficulties, especially intracorporeal gastroduodenostomy. Understanding the comparative effectiveness of laparoscopic and open approaches to resection of early-stage gastric cancer is an important issue in the surgical management of this condition [3]. Laparoscopic approach has developed tremendously especially with the improvement of new endoscopic staplers which make intracorporeal anastomosis feasible \& much easier than before.
Also, laparoscopic procedures have gained a vast reputation in the world where all fields of surgery had increase experience in the laparoscopic procedures \& well- experience laparoscopic surgeons too. The safety and efficacy of laparoscopic gastrectomy in the treatment of early gastric cancer have been demonstrated in many clinical studies [8-11]. Moreover, the benefits of laparoscopic procedures overweighed those of open procedures. Such as, less post-op pain \& analgesia requirement, less blood loss, small wounds, faster recovery \& earlier return to normal bowel function \& oral intake, less hospital stay and early return to work.

In this study, we studied Total Laparoscopic Distal gastrectomy(TLDG) compare to open distal gastrectomy procedures(ODG) where 100 cases from each group compared in different outcome measures. This study aimed to elicit the efficacy \& the feasibility of this procedure compare to the open procedure to the world in presence of qualified laparoscopic surgeon.

Correspondence to: Alhadhrami B, Royal Hospital, Muscat, Oman, Tel: +968 24 599000; E-mail: drbader85@hotmail.com

Received: November 01, 2016; Accepted: November 09, 2016; Published: November 14, 2016 


\section{Methods}

In this single institute, namely East National Cancer centre data were collected retrospectively (after IRB approval i.e. Ethical approval in the institute by the HOD of gastric division. Prof. Takahiro Kinoshita) for all patients who underwent total laparoscopic distal (B1) gastrectomy (TLDG) for early gastric cancer between Aug 2010 and Jul 2012. This procedure was compared to open distal gastrectomy (ODG) of early gastric cancer patients in the same institute between Aug 2008 and Jul 2010. In each group, we reviewed 100 patients. The two groups were case matched in retrospective study analysis in which different parameters were compared. The main parameters of comparison in our study are Operation time, Blood loss, number of harvested lymph node, postoperative hospital stay, clinical pathway, postoperative complications. Each Group has same number of males and females respectively which is 62 males in each group and 38 females and the total of 200 patients. All patients between Aug 2008 and Jul 2010 were done as open procedures because of no availability of laparoscopic surgeon in gastric division in this institute. The other group underwent laparoscopic procedure when laparoscopic surgeon has become available. So, there was no randomization of the two groups as any patient with early gastric cancer was fit for laparoscopy procedure was included between Aug 2010 and Jul 2012. The inclusion criteria for early gastric cancer are satges Ia, Ib and IIa according to the Japanese classification of gastric carcinoma [12]. The patients stage also were matched in number and we chose same number of patients in each stage as it was available for comparison purposes. Also, other parameters were recorded for each patient such as: age, BMI \& Clinical course of oral intake. The height and weight of patients were taken at the admission for surgery. The clinical course defined by $1^{\text {st }}$ Postoperative day (POD) water, $3^{\text {rd }}$ POD meals $\& 8^{\text {th }}$ POD discharge from hospital. The meals were similar for all patients in both groups and it was started at $3^{\text {rd }}$ day post-op and if patient had open bowel by passing flatus or motion. The patient was ready for discharge if he has no vomiting, abdominal pain or fever and capable to take up to $60 \%$ of the meal. All patients were fit for General anaesthesia with ASAI with no comorbidities. Preoperative assessment for all patients was by upper GI series, esophago gasteroendoscopy and Computerized tomography of the chest, the abdomen and Pelvis to stage the tumours according to the Japanese classification of gastric carcinoma. The type of reconstruction for the laparoscopic procedure was Intracorporeal gastroduodenostomy Delat-shaped anastomosis which was accomplished by linear staplers for all patients, this technique was which was introduced by Kanaya, et al. [13]. The open procedure gasteroduodenostomy anastomosis was accomplished by hand-sewn technique in the conventional method.

\section{Surgical technique total laparoscopic billroth-1 gastrec- tomy (using the delta-shaped anastomosis)}

The patient was placed in the modified lithotomy position. Where the surgeon stood at the right side of the patient, first assistant at the left side of the patient and the laparoscopist between the patient's legs. Then the placement of five trocars, 12-mm trocar was inserted through an umbilical incision either with open technique or with optical port, and four other trocars (two $12-\mathrm{mm}$ trocars and two 5-mm trocars) were placed under laparoscopic guidance as shown in the Figure 1. Laparoscopic procedures were performed under a pneumoperitoneum with $10 \mathrm{mmHg} \mathrm{CO}_{2}$. Mobilization of the stomach and end block systematic lymph node dissection was performed laparoscopically. The duodenum was transacted using an endoscopic linear stapler during lymph node dissection. Preservation of the vagal nerve system was not performed.
The resection line of the stomach was determined with guidance from intraoperative gastroendoscopy, which recognized the marking clips that were placed preoperatively. The stomach was resected using an endoscopic linear stapler from the greater curvature site. The resected stomach was extracted via a $3-\mathrm{cm}$ incision at the umbilical port (Figure 1 ), and the pneumoperitoneum was re-established. A small hole was made on the greater curvature side of the stomach and the posterior side of the duodenal stump. A 45-mm endoscopic linear stapler was introduced through a left lower port, and each jaw was inserted in the created holes. The stapler was closed and fired, thus attaching the posterior wall of the remnant stomach and the duodenum (Figure 2A). The entry hole was closed using two applications of the endoscopic. Linear stapler assisted by three stay sutures placed in advance (Figure $2 \mathrm{~B}$ and $2 \mathrm{C}$ ). The gastroduodenostomy was immersed in normal saline and tested for leaks by infusing air into the pouch lumen via a nasogastric tube and looking for escaping bubbles [3].

\section{Results}

In this study the values are the means $\pm \mathrm{SD}$. The $p$-value less than 0.05 was considered as significant The Student's t-test or the chi-square test were used to test for the comparisons among groups.

A total of 200 patients were included in this study. Each group include 100 patients as case-matched analysis. Characteristics of the patients were almost similar. We reviewed same number of males to female ratio in both groups which was 62:38. For the age in the TLDG group was $62.9( \pm 11.1)$ and in the ODG group was $65.4( \pm 10.7)$ for ODG. The body mass index (BMI) the TLDG group was $22.5( \pm 2.6)$ and for ODG was $22.6( \pm 3)$ Table 1.

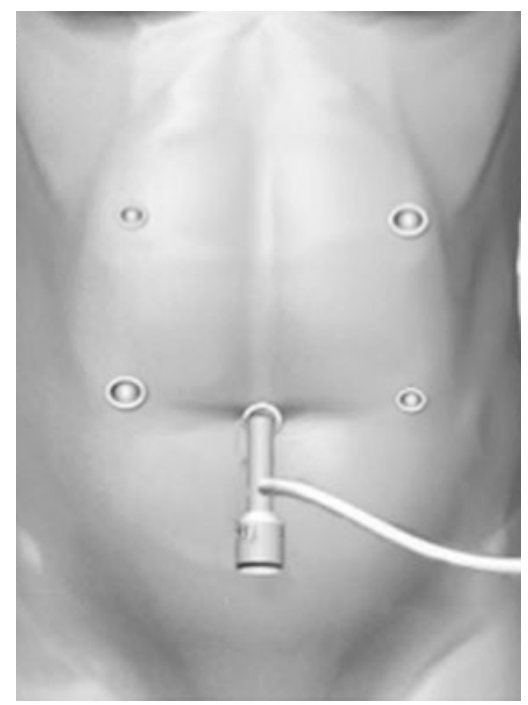

Figure 1. Laparoscopic procedure.

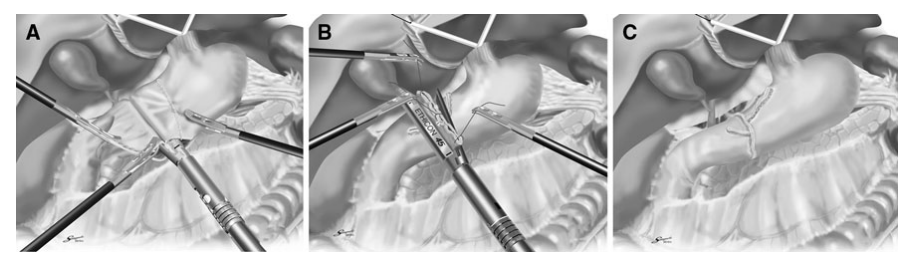

Figure 2. Schematic outlines of the intracorporeal gastroduodenostomy (delta-shaped anastomosis). A) The posterior wall of the remnant stomach and the duodenum are attached with an endoscopic linear stapler. B) The entry hole is closed with the endoscopic linear stapler with the assistance of three stay sutures placed in advance. C) After completion of the anastomosis. 
The Early gastric cancer was considered for any patient with stage $1 \mathrm{a}, 1 \mathrm{~b} \& 2 \mathrm{a}$. We took same number of patients in each group of tumor stages. There were 79 patients with stage I A, 17 patients with stage I B and 4 patients with stage II A in both groups table 2 .

There were significant differences $(P<0.001)$ present between the two groups in blood loss, operation time \& postoperative hospital stay. For the estimated blood loss was significantly less for the TLDG group which was $17.9( \pm 21.8) \mathrm{ml}$ and for the ODG was $234( \pm 257.7) \mathrm{ml}$. Operation time was longer for the Open group which was $204( \pm 42.7)$ and for TLDG was 173 $( \pm 30.5) \mathrm{min}$. The Post-operative hospital stay was for TLDG was $8.5( \pm 2.4)$ Days and for the ODG was $11.2( \pm 9.5)$ Table 3.

The clinical recovery was fester in the TLDG group which was $95 \%$ and $87 \%$ in the ODG group but it was not statistically significant. In the TLDG group $98 \%$ could take more than $60 \%$ of their daily meal at the day of discharge compare to $95 \%$ in the ODG group. Also, this was not statistically significant. There were 2 cases of bleeding from the stapler line postoperatively in the TLDG group managed endoscopically and one case in the ODG which was managed conservatively. There was 1 case of leak in the TLDG group and 2 cases in the ODG group. There was no patient has stenosis in the follow-up Table 4.

The extent of lymph node dissection was similar between the two groups with no Significance over all as shown in Table 5.

\section{Discussion}

Many advantages may be attributed to laparoscopic gastric surgery, such as better cosmetic effect, improved quality of life, less intense pain, shortened hospital stay, early rehabilitation, and early return to social activity. There are many reports have demonstrated these significant benefits and its less morbidity even in elderly patients [14-20]. Also, some other reports concluded that laparoscopic gastrectomy is a

Table 1. Characteristics of patients.

\begin{tabular}{|l|c|c|c|}
\hline & TLDG $(\mathbf{n}=\mathbf{1 0 0})$ & ODG $(\mathbf{n}=\mathbf{1 0 0})$ & p-value \\
\hline $\mathrm{M} / \mathrm{F}$ & $62 / 38$ & $62 / 38$ & \\
\hline Age & $62.9( \pm 11.1)$ & $65.4( \pm 10.7)$ & 0.139 \\
\hline BMI & $22.5( \pm 2.6)$ & $22.6( \pm 3)$ & \\
\hline
\end{tabular}

Table 2. Patients tumor stage.

\begin{tabular}{|c|c|c|}
\hline Stage & TLDG $(\mathbf{n}=\mathbf{1 0 0})$ & ODG $(\mathbf{n}=\mathbf{1 0 0})$ \\
\hline I A & 79 & 79 \\
\hline I B & 17 & 17 \\
\hline II A & 4 & 4 \\
\hline
\end{tabular}

Table 3. Surgical outcomes.

\begin{tabular}{|l|c|c|c|}
\hline \multicolumn{1}{|c|}{ TLDG } & ODG & $\boldsymbol{P}$-value \\
\hline Blood Loss & $17.9( \pm 21.8) \mathrm{ml}$ & $234( \pm 257.7) \mathrm{ml}$ & $P<0.001^{*}$ \\
\hline Operation time & $173( \pm 30.5) \mathrm{min}$ & $204( \pm 42.7) \mathrm{min}$ & $P<0.001^{*}$ \\
\hline Hospital stay & $8.5( \pm 2.4)$ days & $11.2( \pm 9.5)$ days & $P<0.001^{*}$ \\
\hline
\end{tabular}

*Statistical significance.

Table 4. Post-operative course.

\begin{tabular}{|l|c|c|c|}
\hline & TLDG & ODG & p-value \\
\hline Clinical Course(recovery) & $95 \%$ & $87 \%$ & 0.119 \\
\hline Discharge at $>60 \%$ Of Meal & $98 \%$ & $95 \%$ & 0.251 \\
\hline Complication & & & \\
\hline Leak & 1 & 2 & \\
\hline Stenosis & 0 & 0 & \\
\hline Bleeding & 2 & 1 & \\
\hline
\end{tabular}

feasible and safe procedure when performed by qualified experienced laparoscopic surgeon [21-23]. The Billroth-1 Gastrectomy is a very useful reconstruction technique when indicated. It allows the physiological passage of diet through the duodenum but it sometimes can cause bile regurgitation resulting in biliary esophagitis. However, the technique is commonly performed for early gastric cancer especially it allow easy post-operative surveillance and follow-up with endoscopy compared to other techniques of reconstruction like Roux-y [24-26].

Early gastric cancer(EGC) is a term originated in Japan in 1962. EGC is defined as gastric cancer which is invasive and restricted to the mucosa and submucoas irrespective of lymph node metastasis [27].

Total laparoscopic distal Gastrectomy is at this institute the main procedure at present where $70 \%$ cases done laparoscopically and $30 \%$ done in open procedure only. The early gastric cancer exclusively done by laparoscopy with D1+ or D2 lymph node dissection as indicated.

In this study early gastric cancer was considered for Ia,Ib \& IIa as shown in table 6 .

Lymph node dissection extent in our cases were between D1+ \& D2. In Japan D2 dissection had been accepted as standard lymphnode dissection when indicated according to the Japanese classification. Also, our study reported no satistacical difference in number of lymp nodes harveseted all over, except for No. 1 which was more good harvesment achieved by laparoscopy.

In this study we tried to match the cases in both group. The age, gender, BMI were almost similar with no statistical difference. Also, same number of patients of tumour stages were included. Even though many cases with advanced Gastric cancer also done laparoscopically done in the institute we did not include them as they require total gastrectomy or proximal gastrectomy which need highly advance laparoscopic skills and learning curve. Also, still not widely accepted like the case in early gastric cancer.

Table 5. Number of lymphnodes.

\begin{tabular}{|c|c|c|c|}
\hline $\begin{array}{c}\text { Anatomical definitions of } \\
\text { lymph node stations(15) }\end{array}$ & TLDG(SD) & ODG(SD) & p-value (SD) \\
\hline 1 & 3.96 & 2.99 & 0.002 \\
\hline $3 \mathrm{a}$ & 3.30 & 3.88 & NS \\
\hline $3 \mathrm{~b}$ & 1.13 & 0.78 & NS \\
\hline $4 \mathrm{sb}$ & 1.36 & 1.69 & NS \\
\hline $4 \mathrm{~d}$ & 5.21 & 5.14 & NS \\
\hline 5 & 0.53 & 0.62 & NS \\
\hline 6 & 4.46 & 4.26 & NS \\
\hline 7 & 2.21 & 2.18 & NS \\
\hline $8 \mathrm{a}$ & 2.96 & 3.17 & $\mathrm{NS}$ \\
\hline 9 & 2.24 & 2.31 & $\mathrm{NS}$ \\
\hline $11 \mathrm{p}$ & 2.05 & 1.88 & $\mathrm{NS}$ \\
\hline $12 \mathrm{a}$ & 1.00 & 0.79 & $\mathrm{NS}$ \\
\hline Total & 30.6 & 31.2 & $\mathrm{NS}$ \\
\hline
\end{tabular}

Table 6. Gastric cancer was considered for Ia,Ib \& IIa.

\begin{tabular}{|c|c|c|c|l|}
\hline & N0 & N1 & N2 & N3 \\
\hline T1a(M), T1b(SM) & IA & IB & IIA & IIIB \\
\hline T2(MP) & IB & IIA & IIB & IIIA \\
\hline T3(SS) & IIA & IIB & IIIA & IIIB \\
\hline T4a(SE) & IIB & IIIA & IIIB & IIIC \\
\hline T4b(SI) & IIIB & IIIB & IIIC & IIIC \\
\hline M1 (Any T, Any N) & & \multicolumn{3}{|l}{} \\
\hline
\end{tabular}


We found in this study that TLDG compare ODG with statistic significance that blood loss is much less in the laparoscopic group which is very important in dealing with cancer patients. Who can have anemia as common lab test findng. Also, we found operation time is less in the laparoscopic group compare to the open group. We believe this is an important point for laparoscopic surgeon when concerning major procedures laparoscopically. In this study we prove with statistic significance that it is possible when qualified experienced surgeon present. Our study showed that postoperative hospital stay was much in the laparoscopic group compared to the open group with statistic significance. In the laparoscopic group the hospital stay was around 8 days with slight variations between the patients. Unlike the open group which was around 11 days with large variation up to 9 days.

There were no patients with wound complications, nevertheless the wounds healing were much faster in the TLDG and cosmetically more accepted to the patients compare to lapartomy wound.

For the world wide consideration of total laparoscopic distal gastrectomy we recommend laparoscopic surgeons to have training before adopting laparoscopic gastrectomy in their institutes in like animal laps or virtual simulators and or invitation of an experienced laparoscopic surgeon to their institute. The procedure is easily can be learned and the learning curve is short. Training courses focusing on intensive explanations are needed for team building which may be now available by many surgical societies around the globe. Also, we recommend starting with early gastric cancer patients who are with status of ASAI.

One of the important concerns of intracorporeal anastomosis is determination of the resection line of the stomach, which maintains sufficient proximal margins. Because in early gastric cancer, which cannot be recognized from the serosal side and cannot be felt by the surgeon's hand during intracorporeal procedures. To resolve this problem, we placed marking clips at the oral side of the lesion before surgery and applied intraoperative gastroscopy to confirm them. Indeed, in our series, we did not detect cancer positive tissue at the proximal margin.

In delta-shaped anastomosis, gastroduodenostomy is a combination of introverted and extroverted linear stapling, which may result in a larger lumen that is not limited by the duodenal lumen [3]. We believe that closing of the gastroduodenostomy stump is the only technically demanding for this procedure as it requires from the laparoscopic surgeon the capability of laparoscopic suturing technique but we think it is not difficult with training especially in dry box. In this study, there are some limitations that it is reflect the experience of single institute. We think multi-center study perspective stud will show more realistic and more statistically representative. Also, in this study the costs of the procedure were not studied as this issue can be very important in some countries with low economic status and affect their acceptance to the procedure. Also, the requirement of blood transfusion was not mention when comparing between the two groups.

In conclusion, our study give a statistical significant evidence that total laparoscopic distal gastrectomy has a better surgical outcomes when compared with open distal gastrectomy. Laparoscopic B I gastrectomy in early gastric cancer is feasible and a safe technique in the presence of experienced laparoscopic surgeon. World-wide spread of this technique can be accomplishing with the help of advance laparoscopic courses.

\section{Disclosures}

No disclosures

\section{References}

1. http://www.cancerresearchuk.org

2. Ikeda T, Kawano H, Hisamatsu Y, Ando K, Saeki H, et al. (2013) Progression from laparoscopic-assisted to totally laparoscopic distal gastrectomy: comparison of circular stapler (i-DST) and linear stapler (BBT) for intracorporeal anastomosis. Surg Endosc 27: 325-332. [Crossref]

3. Kinoshita T, Shibasaki H, Oshiro T, Ooshiro M, Okazumi S, et al. (2011) Comparison of laparoscopy-assisted and total laparoscopic Billroth-I gastrectomy for gastric cancer: a report of short-term outcomes. Surg Endosc 25: 1395-1401. [Crossref]

4. Adachi Y, Suematsu T, Shiraishi N, Katsuta T, Morimoto A, et al. (1999) Quality of life after laparoscopy-assisted Billroth I gastrectomy. Ann Surg 229: 49-54. [Crossref]

5. Huscher CG, Mingoli A, Sgarzini G, Sansonetti A, Di Paol M, et al. (2005) Laparoscopic versus open subtotal gastrectomy for distal gastric cancer: five-year results of a randomized prospective trial. Ann Surg 241: 232-237

6. Kitano S, Shiraishi N, Fujii K, Yasuda K, Inomata M, et al. (2002) A randomized controlled trial comparing open vs laparoscopy-assisted distal gastrectomy for the treatment of earlygastric cancer: an interim report. Surgery 131(1 Suppl): S306-S311. [Crossref]

7. Yasunaga H, Horiguchi H, Kuwabara K, Matsuda S, Fushimi K, et al. (2012) Annals of surgery.

8. Uyama I, Sugioka A, Matsui H, Fujita J, Komori Y, et al. (2000) Laparoscopic D2 lymph node dissection for advanced gastric cancer located in the middle or lower third portion of the stomach. Gastric Cancer 3: 50-55. [Crossref]

9. Tanimura S, Higashino M, Fukunaga Y, Takemura M, Tanaka Y, et al. (2005) Laparoscopic gastrectomy for gastric cancer: experience with more than 600 cases. Surg Endosc 19: 1177-1181.

10. Shiraishi N, Yasuda K, Kitano S (2006) Laparoscopic gastrectomy with lymph node dissection for gastric cancer. Gastric Cancer 9: 167-176. [Crossref]

11. Kim MC, Kim KH, Kim HH, Jung GJ (2005) Comparison of laparoscopy-assisted by conventional open distal gastrectomy and extraperigastric lymph node dissection in early gastric cancer. J Surg Oncol 91: 90-94. [Crossref]

12. Japanese Gastric Cancer Association (2011) Japanese classification of gastric carcinoma: 3rd English edition. Gastric Cancer 14: 101-112. [Crossref]

13. Kanaya S, Gomi T, Momoi H, Tamaki N, Isobe H, et al. (2002) Delta-shaped anastomosis in totally laparoscopic Billorth I gastrectomy: new technique of intraabdominal gastroduodenostomy. J Am Coll Surg 195:284-287.

14. Goh PM, Khan AZ, So JB, Lomanto D, Cheah WK, et al. (2001) Early experience with laparoscopic radical gastrectomy for advanced gastric cancer. Surg Laparosc Endosc Percutan Tech 11: 83-87. [Crossref]

15. Adachi Y, Suematsu T, Shiraishi N, Katsuta T, Morimoto A, et al. (1999) Quality of life after laparoscopy-assisted Billroth I gastrectomy. Ann Surg 229: 49-54. [Crossref]

16. Kitano S, Adachi Y, Shiraishi N, Suematsu T, Bando T (1999) Laparoscopic-assisted proximal gastrectomy for early gastric carcinomas. Surg Today 29: 389-391. [Crossref]

17. Hwang SI, Kim HO, Yoo CH, Shin JH, Son BH (2009) Laparoscopic-assisted distal gastrectomy versus open distal gastrectomy for advanced gastric cancer. Surg Endosc 23: 1252-1258. [Crossref]

18. Kunisaki C, Makino H, Takagawa R, Oshima T, Nagano Y, et al. (2009) Efficacy of laparoscopy-assisted distal gastrectomy for gastric cancer in the elderly. Surg Endosc 23: 377-383. [Crossref]

19. Mochiki E, Ohno T, Kamiyama Y, Aihara R, Nakabayashi T, et al. (2005) Laparoscopyassisted gastrectomy for early gastric cancer in young and elderly patients. World $J$ Surg 29: 1585-1591. [Crossref]

20. Koeda K, Nishizuka S, Wakabayashi G (2011) Minimally invasive surgery for gastric cancer: the future standard of care. World J Surg 35: 1469-1477. [Crossref]

21. Yasuda K, Sonoda K, Shiroshita H, Inomata M, Shiraishi N, et al. (2004) Laparoscopically assisted distal gastrectomy for early gastric cancer in the elderly. $\mathrm{Br}$ J Surg 91:1061-1105.

22. Cho GS, Kim W, Kim HH, Ryu SW, Kim MC, et al. (2009) Multicentre study of the 
Alhadhrami B (2016) Total laparoscopic billroth-1(Distal) gastrectomy compare(TLDG) To open billroth-1 gastrectomy(ODG) in early gastric cancer

safety of laparoscopic subtotal gastrectomy for gastric cancer in the elderly. Br J Surg 96: 1437-1442. [Crossref]

23. Kim EJ, Seo KW, Yoon KY (2012) Laparoscopy-assisted distal gastrectomy for early gastric cancer in the elderly. J Gastric Cancer 12: 232-236. [Crossref]

24. Hintze RE, Adler A, VeltzkeW, Abou-Rebyeh H (1997) Endoscopic access to the papilla of Vater for endoscopic retrograde cholangiopancreatography in patients with Billroth II or Roux-en-Y gastrojejunostomy. Endoscopy 29: 69-73.
25. Kojima K, Yamada H, Inokuchi M, Kawano T, Sugihara K (2008) A comparison of Roux-en-Y and Billroth-I reconstruction after laparoscopy-assisted distal gastrectomy. Ann Surg 247: 962-967. [Crossref]

26. Itoi T, Ishii K, Sofuni A, Itokawa F, Tsuchiya T, et al. (2010) Single-balloon enteroscopy-assisted ERCP in patients with Billroth II gastrectomy or Roux-en-Y anastomosis (with video). Am J Gastroenterol 105: 93-99. [Crossref]

27. Gotoda T (2006) Endoscopic resection of early gastric cancer: the Japanese perspective. Curr Opin Gastroenterol 22: 561-569. [Crossref]

Copyright: (C2016 Alhadhrami B. This is an open-access article distributed under the terms of the Creative Commons Attribution License, which permits unrestricted use, distribution, and reproduction in any medium, provided the original author and source are credited. 